\title{
Material Development for ArF Immersion Extension towards Sub-20nm Node
}

\author{
Tsuyoshi Furukawa, Kousuke Terayama, Takeo Shioya and Motoyuki Shima \\ Fine Electronic Research Laboratories \\ Semiconductor Materials Laboratory \\ JSR Corporation \\ 100, Kawajiri-cho, Yokkaichi Mie 510-8552 Japan
}

\begin{abstract}
Today ArF immersion processes are widely applied for advanced device manufacturing. ArF immersion lithography enables 4x nm half-pitch (hp) lithography. The use of double patterning extends immersion to $2 \mathrm{x} \mathrm{nm}$ hp lithography. New materials for such $193 \mathrm{~nm}$ lithography extensions are widely needed, especially for post-193nm immersion lithography technology. In this paper, we report a slimming material that makes line patterns smaller and it succeeds in forming $20 \mathrm{~nm}$ lines.

In another study, we examined a new hydrophilic lactone to improve line width roughness (LWR) and defectivity. First, we studied the lactone reactivity with developer and its behavior during development. Then we verified its line width LWR performance and defectivity. When the hydrophilic new lactone is used in a photoresist, an improvement in LWR and defectivity photoresist was observed. This is very useful progress, especially in combination with processes such as multiple patterning to extend ArF immersion to sub-20nm hp dimensions.
\end{abstract}

Keywords: ArF immersion, 193nm lithography extension, Defectivity, LWR

\section{Introduction}

ArF immersion lithography can extend optical lithographic resolution down to $3 \mathrm{xnm} \mathrm{hp}$. Double patterning techniques can further extend the optical lithography limit to $2 \mathrm{x} \mathrm{nm} \mathrm{hp}[1,2]$. Multiple patterning extends ArF immersion lithography towards sub-20nm hp processes.

Extreme ultraviolet (EUV) lithography has been viewed as the next lithographic method for next generation lithography (NGL). Industries have been anticipating its implementation for high volume device manufacturing. However, there are still many issues to overcome for EUV. Exposure tool readiness, mask manufacturing and photoresist performance are the three main areas of difficulties [3]. Also, its high running cost and operation processes need to be carefully considered and assessed. Since such difficulties remain on EUV process, alternative NGL candidates are being explored and studied.

Directed self-assembly (DSA) is one potential NGL process. DSA is a process that utilizes chemical properties such as polymer molecule cohesion and modeling special block polymers. Pattern formation due to annealing time and the intrinsic pitch limitation of the polymer structures are some reasons why we cannot apply DSA for device manufacturing process today. [4-9]

\begin{tabular}{lll}
\hline Received & May & 19,2013 \\
Accepted & June & 10,2013
\end{tabular}




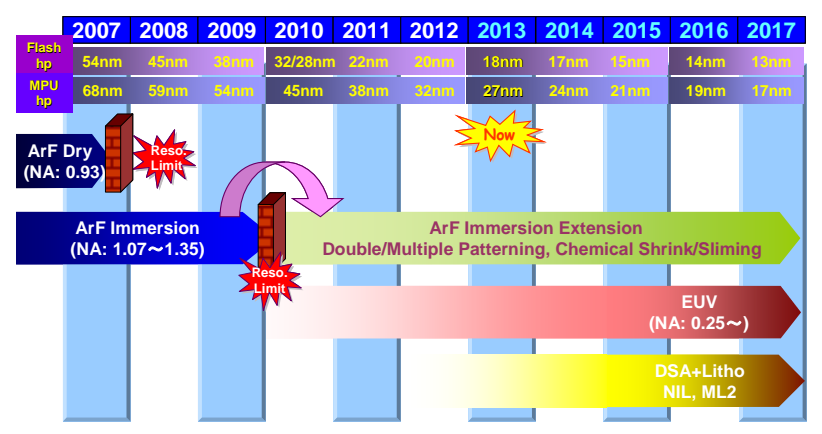

Figure1. Lithography technology trends.

The potential for DSA to extend resolution limit is high. But there are a number of issues to be resolved before moving to the device manufacturing phase, such as shortening annealing times, chemical structure optimization and defect control. Because of these limitations, ArF immersion extension has become the front-up lithographic approach to enable sub-20nm hp requirements.

Any process tricks with ArF immersion and double patterning process are needed for developing sub-20nm hp patterning material. All possible additional processes are being applied to actual, on-wafer photoresist patterns formed by $193 \mathrm{~nm}$ immersion. It is critical to improve the pattern quality of the original $193 \mathrm{~nm}$ resist patterns. Therefore, the pattern quality of the single exposure ArF lithography process, such as defectivity, resolution and LWR are becoming more and more important.

In this paper, we studied not only process tricks, but also improvements to defectivity and LWR. We developed new slimming materials to reduce the critical dimension of line and/or pillar patterns. The material design and performances of these materials are reported in this paper. Also, we focused on photoresist development capable of demonstrating good performance for sub-40nm hp resolution with well controlled LWR and defectivity.

\section{Experimental}

\subsection{Slimming Process}

\subsubsection{Materials and Process}

JSR NFC S310 was used as slimming material. JSR $193 \mathrm{~nm}$ photoresist, a methacrylate base $193 \mathrm{~nm}$ photoresist, was used throughout this study. JSR SOG (spin on glass) and SOC (spin on carbon) were spin-coated on substrates as underlayer of slimming process. Tetramethylammonium hydroxide (aqueous, 2.38 wt \%) was used to develop the photoresist. Deionized water (DIW) was used for immersion exposures.

Our line slimming process was implemented using the following process flow. Slimming material was coated directly onto the photoresist pattern at $1500 \mathrm{RPM}$. Then, the $80 \mathrm{C} / 60 \mathrm{C}$ bake process was applied for the reaction to occur between photoresist and slimming material. Reacted photoresist and slimming material was removed by TMAH to obtain the slimmed photoresist patterns.

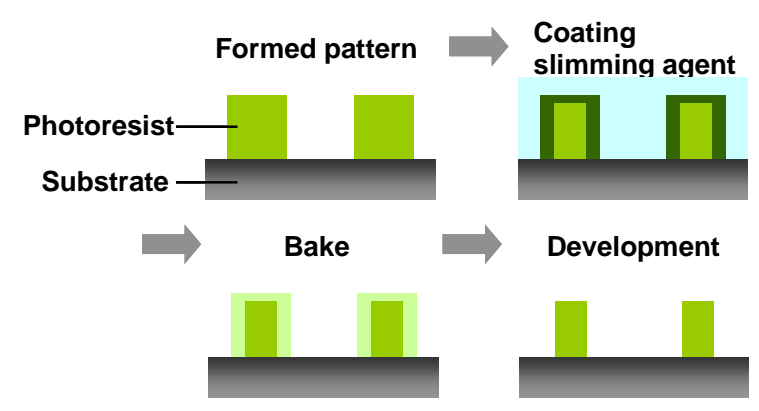

Figure2. Photoresist pattern slimming.

\subsubsection{Evaluation tools}

A CLEAN TRACK LITHIUS Pro-i (TEL) or CLEAN TRACK ACT12 (TEL) was used for the photoresist / SiARC / SOC coating, bake and development processes. Exposure was carried out with a Nikon S610 (NA 1.30). Etching was done with a Tellius SCCM (TEL). CD measurement and observation of top-down profile for each wafer was performed using the CD-SEM: CG-4000 (Hitachi High-Tech.). Cross-section profile was observed using the X-SEM S4800 (Hitachi High-Tech).

Etching steps were implemented under the following conditions: SOG etching condition was $\mathrm{CF}_{4}=120 \mathrm{sccm}$, Press. $=80 \mathrm{mTorr}, \mathrm{RF}$ top $/$ bottom $=300 \mathrm{~W} / 300 \mathrm{~W}$. UDL etching condition was $\mathrm{O}_{2} / \mathrm{N}_{2}$ $=55 / 65 \mathrm{sccm}, \quad$ Press $=10 \mathrm{mTorr}, \quad \mathrm{RF}$ top $/$ bottom $=700 \mathrm{~W} / 100 \mathrm{~W}$. Silicon oxide etching condition was $\mathrm{CHF}_{3} / \mathrm{Ar} / \mathrm{O}_{2}=90 / 400 / 10$ sccm, $\quad$ Press. $=100 \mathrm{mTorr}$, $\mathrm{RF}$ top $/$ bottom $=400 \mathrm{~W} / 800 \mathrm{~W}$.

2.2. LWR and defectivity improvement by applying a new lactone

\subsubsection{Materials}

The structures of polymer in this paper are shown in Figure3. 


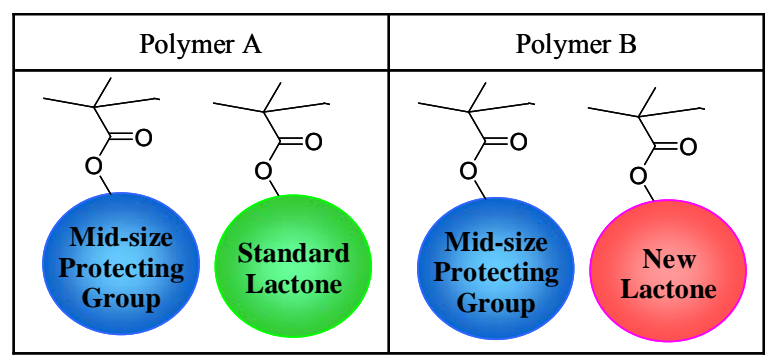

Figure3. Polymer structures.

Photoresist sample for this lactone study were obtained by mixing polymer, PAG, quencher and solvent. The mixed solution was filtered by $0.02 \mathrm{um}$ PTFE filter prior to general analysis and litho performance evaluation. Regarding defectivity evaluation samples, the mixed solution was circulated and filtered with $0.01 \mathrm{um}$ Nylon filter and $0.005 \mathrm{um}$ HDPE filter. The photoresist formulations are described in Table 1.

Table1. Photoresist formulations.

\begin{tabular}{|c|c|c|c|}
\hline Resist & Polymer & PAG & Quencher \\
\cline { 1 - 2 } Resist A & Polymer A & \multirow{2}{*}{ Common } & \multirow{2}{*}{ Common } \\
\cline { 1 - 2 } Resist B & Polymer B & & \\
\hline
\end{tabular}

2.2.2. Investigation of lactone hydrophilicity and reactivity with developer

Static contact angle measurement was carried out with Kruss DSA10L2E. Wafers were prepared with the following procedure before development wafer preparation, photoresist sample was spin coated to $100 \mathrm{~nm}$ thickness after a $100{ }^{\circ} \mathrm{C} 60$ sec bake on a HMDS-treated bare silicon wafer. After development wafer preparation: photoresist sample was spin coated at $100 \mathrm{~nm}$ thickness after a $100^{\circ} \mathrm{C} 60 \mathrm{sec}$ bake on a HMDS-treated bare silicon wafer. After that, the wafer was developed with tetramethylammonium hydroxide aqueous (TMAH $2.38 \mathrm{wt} \%$ ).

\subsubsection{Litho performance evaluation condition}

Photoresist exposure was done using a Nikon NSR-S610C ArF immersion exposure tool. Observation of the top down photoresist profile was carried out with a Hitachi High-Technologies CG4100 SEM. The LWR was defined as $3 \sigma$ of the resist line width distribution.

\subsubsection{Defectivity evaluation conditions}

Photoresist exposure was done using a Nikon NSR-S610C. Defect inspection was carried out with a KLA-Tencor KLA2810. Observation of defect images was carried out with Hitachi High-Technologies multi purpose SEM RS6000.

\section{Results and Discussion}

\subsection{Slimming process}

In this process, semi-dense small line width patterns are used as core templates. Therefore, one of the most critical processes is the formation of such small patterns. Semi-dense lines with a small line width CD are prone to line collapse and are well known to demonstrate narrow process windows. Therefore, we propose formations of such small line width semi-dense patterns using two steps. First, line patterns with 10 to $20 \mathrm{~nm}$ wider lines are formed. This process improved the process window of the lines by a sizeable amount. Then, these patterns are reduced using our slimming agent, FZX S310, to form line patterns of the final targeted line width. In this slimming process, wider process window was observed compared to an over-dose process. And the pillar slimming process resulted in almost identical characteristics as the original pattern. The slimming amount of pillar condition is over $30 \mathrm{~nm}$. Generating such small pillar patterns of $30 \mathrm{~nm}$ size using an over-exposure condition showed no process window. However, by using the slimming process, we achieved a wider process window. So, the slimming process is useful for making small lines or pillar patterns.

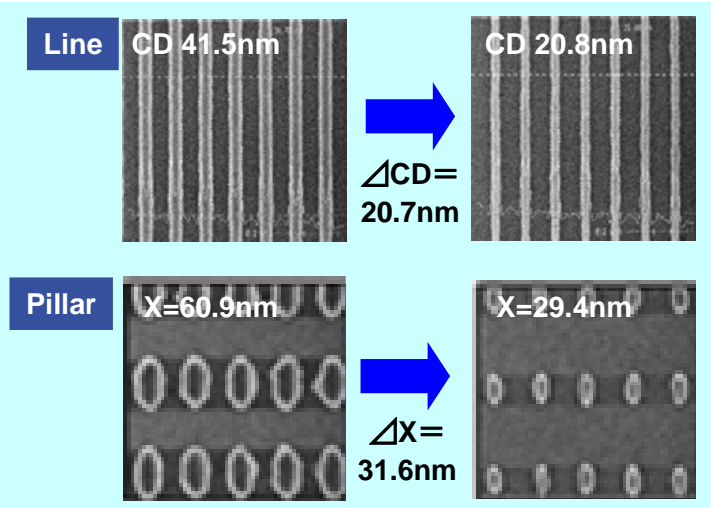

Figure4. Post-slimming results for line and pillar patterns.

In another analogous process, a silicon oxide layer is being used as core pattern for 
silicon nitride CVD deposition. So we investigated the capability of pattern transfer from slimmed line into silicon oxide layer. In this pattern transfer evaluation, photoresist patterns are formed on SOG and SOC layer above silicon oxide wafer. Results of the pattern transfer are summarized step-by-step in Figure5.

\begin{tabular}{|c|c|c|c|}
\hline PR CD & \multicolumn{3}{c|}{ CD=20nm } \\
\hline Tool & CD-SEM & X-SEM \\
\hline $\begin{array}{c}\text { After } \\
\text { PR dev. }\end{array}$ & & & \\
\hline
\end{tabular}

Figure5. Slimmed Pattern transfer into $\mathrm{SiO}_{2}$ layer.

3.2. LWR and defectivity improvement by applying new lactone

We studied a standard lactone and a new lactone monomer which has different hydrophilicity characteristics. The hydrophilicity of each lactone monomer was measured using the retention time of a HPLC measurement; the retention time is described in Table 2.

Table2. HPLC retention time.

\begin{tabular}{|c|c|c|}
\hline Lactone Monomer & Standard \\
\hline Chemical Structure & $\begin{array}{c}\text { Standard } \\
\text { Lactone }\end{array}$ & $\begin{array}{c}\text { New } \\
\text { Lactone }\end{array}$ \\
\hline $\begin{array}{c}\text { Retention Time } \\
\text { at HPLC measurement }\end{array}$ & 4.06 & 3.53 \\
\hline
\end{tabular}

To investigate lactone reactivity with developer, static contact angle measurements were carried out. Figure6 shows the static contact angle of each photoresist before and after development. Photoresist B with the new lactone showed increased hydrophilicity post-development. It indicated that the new lactone unit had higher reactivity compared to the standard lactone.

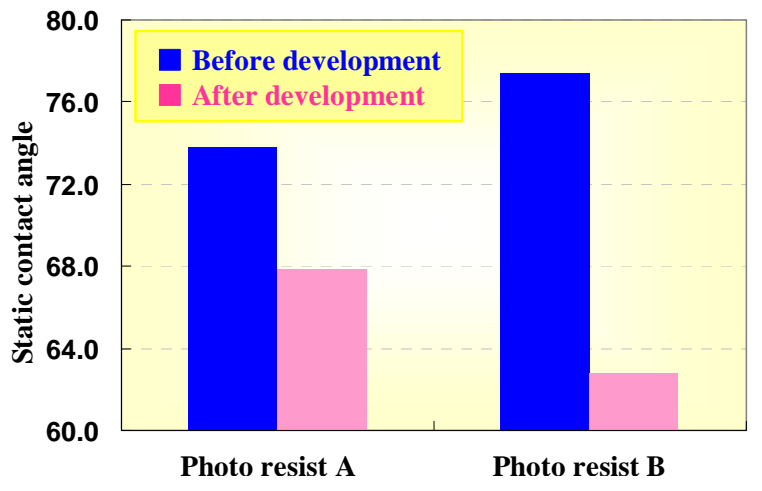

Figure6. Static contact angle before and after development.

QCM measurement was carried out to investigate the behavior during development of each photoresist. As a result, photoresist B did not swell after development compared to photoresist A. It indicated that our new lactone had increased solubility to developer. The result of the QCM measurements is shown in Figure7.

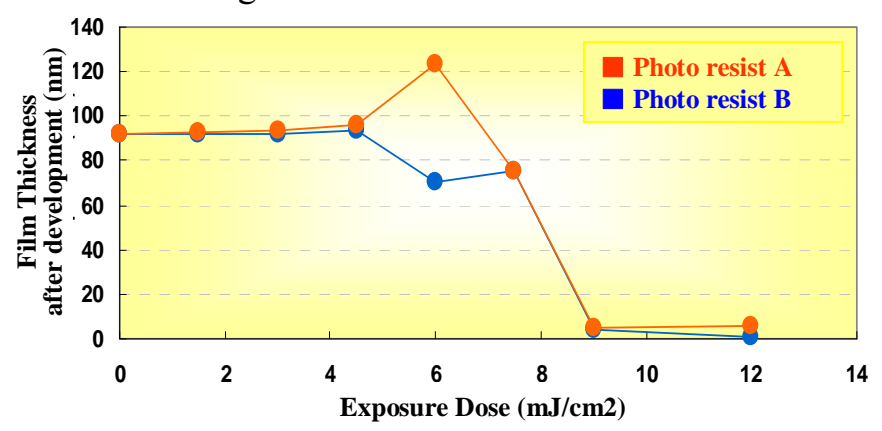

Figure7. QCM measurement.

Dissolution rate measurements were carried out to investigate the photoresist contrast $(\gamma)$ curve. Photoresist B showed higher $\gamma$ value compared to photoresist A. This indicated that the new lactone had higher developer affinity. The result of dissolution rate measurement is shown in Figure8. 


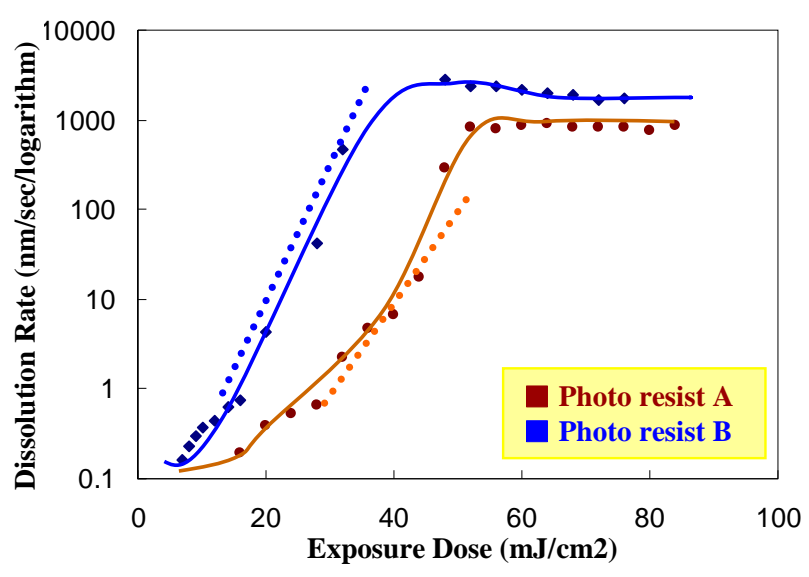

Figure8. Dissolution rates.

LWR measurements were carried out. Figure9 shows the top down profiles of $40 \mathrm{~nm}$ line $100 \mathrm{~nm}$ pitch semi-dense patterns. Resist with the new lactone showed much improved LWR compared to the resist using the conventional lactone.
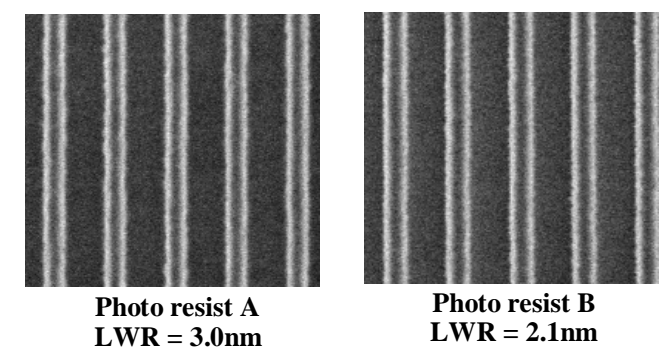

Figure9. Top down pattern profile.

Defectivity inspection was also carried out. Resist using the new lactone showed improved bridge defectivity compared to resist using the conventional lactone. The result of our defectivity evaluation is shown in Figure 10.

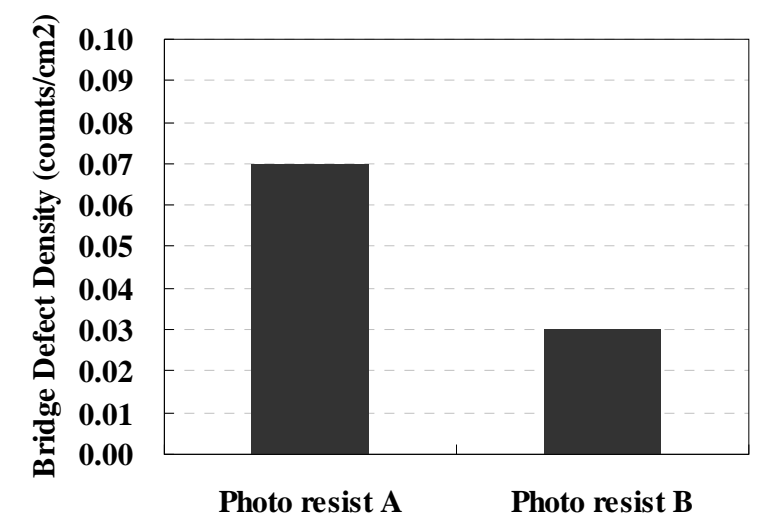

Figure10. Defect density.

\section{Conclusion}

Our experimental data indicated that our slimming process and subsequent pattern transferring process into silicon oxide are applicable for extending ArF immersion technology. We have successfully demonstrated $20 \mathrm{~nm}$ line formation, which could be used as a core pattern. In terms of applying these new materials into high volume manufacturing facilities, further material optimization and process evaluation, such as investigating process stability, is underway.

ArF immersion processes are widely applied for today's advanced device manufacturing. ArF immersion lithography can enable $4 \mathrm{x} \mathrm{nm} \mathrm{hp}$ lithography on its own. It is crucial for materials to maximize the performance of ArF immersion tools and processes.

In this study, we focused on photoresist development to enable sub-40nm hp resolution with well controlled LWR and defectivity.

We studied the effect of lactone polarity and reactivity with developer on lithographic performance and defectivity. We found that the polymer's lactone unit plays a key function for ArF immersion resist performance. A new lactone which showed hydrophilicity and higher reactivity, used in for a photoresist, showed improvement LWR and defectivity compared to a standard lactone-containing resist.

This achievement is very useful for extending existing ArF immersion lithography to the sub-20nm hp regime when utilized in combination with processes such as multiple patterning.

\section{Acknowledgement}

The authors gratefully thank IMEC for their support of these experiments, through the utilization of their tools and facilities.

\section{References}

1. Koji Ito, Michihiro Mita, Goji Wakamatsu, Yusuke Anno, Tomohisa Fujisawa, Hitoshi Osaki, Kenji Hoshiko, Hiromitsu Tanaka, Yukio Nishimura, Makoto Sugiura, Yoshikazu Yamaguchi and Tsutomu Shimokawa, "Non Topcoat Self-freezing Photoresist for Double Patterning Process," J. Photopolym. Sci. Tech., 23 (2010) 199.

2. Tomohiko Kakizawa, Goji Wakamatsu, Yusuke Anno, Masahumi Hori, Michihiro Mita, Kenji Hoshiko, Takeo Shioya, Koichi 
Fujiwara, Shiro Kusumoto, Yoshikazu Yamaguchi and Tsutomu Shimokawa, "Freezing Material Development for Double Patterning Process," J. Photopolym. Sci. Tech., 22 (2005) 641.

3. Toru Kimura, Kouta Nishino, Makoto Shimizu, Yuuki Hirai, Ken Maruyama and Toshiyuki Kai, "JSR EUV Resist Development toward 22nmhp Design and Beyond," J. Photopolym. Sci. Tech., 23 (2010) 643.

4. Hidetami Yaegashi, Kenichi Oyama, Kazuo Yabe, Shoichi Yamauchi, Arisa Hara, Sakuraoka Natori, J. Photopolym. Sci. Tech., 24 (2011) 491

5. Ken Maruyama et all, SPIE Advanced Lithography 2012, Extreme Ultraviolet (EUV) Lithography, Proc. SPIE, 8325 (2012) 8325-10.
6. Daniel P. Sanders, Joy Cheng, Charles T. Rettner, William D. Hinsberg, Ho-Cheol Kim, Hoa Trung, Alexander Fritz, Steven Harrer, Steven Holmes, Matthew Colburn, J. Photopolym. Sci. Tech., 23 (2010) 11.

7. Hiroshi Yoshida, Yasuhiko Tada, Yoshihito Ishida, Teruaki Hayakawa, Mikihito Takenaka, Hirokazu Hasegawa, J. Photopolym. Sci. Tech., 24 (2011) 577.

8. Yoshihito Ishida, Yasuhiko Tada, Tomoyasu Hirai, Raita Goseki, Masa-aki Kakimoto, Hiroshi Yoshida, Teruaki Hayakawa, $J$. Photopolym. Sci. Tech., 23 (2010) 155.

9. Shigeki Hattori, Koji Asakawa, Satoshi Mikoshiba, Hiroko Nakamura, Atsushi Hieno, Yuriko Seino, Mashiro Kanno, Tsukasa Azumam, J. Photopolym. Sci. Tech., 24 (2011) 581 . 